\title{
Effect of secretin and cholecystokinin-pancreozymin extracts on gastric motility in man
}

\author{
L. P. JOHNSON, J. C. BROWN ${ }^{1}$, AND D. F. MAGEE \\ From the Departments of Surgery and Pharmacology, University of Washington, \\ School of Medicine, Seattle, Washington, U.S.A.
}

EDITORIAL SYNOPSIS Extracts of duodenal mucosa containing cholecystokinin and pancreozymin inhibited gastric motility completely in eight subjects whereas secretin extracts produced variable results. It is suggested that cholecystokinin-pancreozymin extracts may contain enterogastrone.

The inhibition of gastric motility induced by placing fat in the duodenum was demonstrated to be humoral by Farrell and Ivy (1926). Subsequent studies on extracts of duodenal mucosa have failed to define the substance involved, although the concept that 'enterogastrone' is a duodenal hormone inhibiting both gastric motility and acid secretion is accepted (Grossman, 1950).

In recent years, specific extracts of duodenal mucosa containing cholecystokinin, secretin, and pancreozymin activity (Jorpes and Mutt, 1959) have been prepared. One of these preparations containing secretin was found to inhibit gastric acid secretion (Greenlee, Longhi, Guerrero, Nelsen, El Bedri, and Dragstedt, 1957). This work was later confirmed with the improved duodenal extracts currently available (Wormsley and Grossman, 1964). Recently extracts containing cholecystokinin-pancreozymin activity were also found to inhibit gastric acid secretion and to exceed secretin preparations in potency (Gillespie and Grossman, 1964; Jordan and De la Rosa, 1965).

In the study of duodenal extracts and their relation to gastric motor activity, it is noteworthy that one of the first preparations used to duplicate the action of fat in the duodenum was an extract containing cholecystokinetic activity (Kosaka and Lim, 1930). Current studies in this laboratory have demonstrated gastric motor inhibition in denervated fundic pouches and innervated stomachs of dogs by intravenous infusions of a cholecystokinin-pancreozymin preparation (Johnson and Magee, 1965). Secretin extracts did not alter gastric motility. It was suggested that the cholecystokinin-pancreozymin preparation contains the physiological activity

${ }^{1}$ Present address: Department of Physiology University of British Columbia, Vancouver 8, B.C., Canada.

2Supported by U.S.P.H.S. Grants AM-02472, AM-05277 and GM 325-05. Wellcome Trust Grant, Burroughs Wellcome and Co. ascribed to enterogastrone and duplicates the action of fat in the duodenum.

The present study tests the effect of two extracts of duodenal mucosa, one containing secretin and the other cholecystokinin-pancreozymin, on gastric motor activity in man.

\section{METHODS}

The study was performed on eight subjects, seven males and one female, between the ages of 20 and 28. All tests were made after an overnight fast. A condom balloon fixed to a plastic levine tube (16F) was passed into the stomach and inflated with $400 \mathrm{ml}$. of air. The tube was then connected to a water-filled, low-pressure Statham transducer. Pressure changes were monitored by a penwriting recorder, the subject lying quietly in the supine position. A slow intravenous infusion of $5 \%$ glucose solution was started in the forearm and continued throughout the experiment. A constant-speed infusion pump was used to infuse the extracts slowly into the intravenous tubing, making it possible to give the preparations without the knowledge of the patient. This was considered important as almost any anxiety-producing stimulus was found to be capable of inhibiting gastric motor function in some subjects.

After a suitable basal recording, a secretin preparation ${ }^{3}$ (hereafter termed 'secretin') was infused over a 10-minute period at $0.05 \mathrm{unit} / \mathrm{kg}$. $/ \mathrm{min}$. to a total dose of $0.5 \mathrm{unit} / \mathrm{kg}$. When gastric activity had returned to normal, a cholecystokinin-pancreozymin preparation $\left(\right.$ Cecekin $\left.^{4}\right)$ was infused in exactly the same manner and dose. Again the motor activity was monitored until it returned to normal.

The eight subjects included in the study experienced no untoward effects from the procedure. Two volunteers were excluded as vomiting occurred in both, one during and the other shortly after, infusion of Cecekin.

The resulting gastric pressure tracings were analysed by comparing a 10-minute control period just before secretin infusion, a 10-minute period during maximum 'Secretin ${ }^{R}$, Vitrum, Stockholm, Sweden.

'CecekinR, Vitrum, Stockholm, Sweden. 
inhibition by secretin or Cecekin, and a 10-minute period during maximum recovery of activity. Several measurements were utilized to compare activity within the three time periods, and these are defined as follows:

1 Changes in mean pressure express the difference in millimetres between the mean point of all waves in the control period with that during maximum inhibition. The mean is established as the mid-point between the highest peak and lowest point of relaxation during the period. Pressure is expressed in millimetres measured from the tracing with each subject serving as his own control. Actual intragastric pressures can be determined from the tracing using a conversion of $10 \mathrm{~mm}$. $=6.4 \mathrm{~cm} . \mathrm{H}_{2} \mathrm{O}$ pressure. The range of intragastric pressures varied from 0 to $48 \mathrm{~cm}$. of water.

2 Latent period is the length of time from the onset of infusion to the beginning of inhibition measured in minutes.

3 Contraction frequency is the number of pressure waves per 10 minutes in the control and infusion periods compared.

4 Percentage activity is the percentage of time during the 10-minute period when pressure wave activity is seen.

5 Amplitude index is the summation of amplitudes of all the waves during the 10-minute period. Amplitudes are measured from the base of each contraction and thus 'amplitude index' expresses only wave height and not changes in baseline.

6 Duration of inhibition is the elapsed time from beginning inhibition to full recovery of activity.

\section{RESULTS}

Tracings from two experiments are shown in Figures 1 and 2. The larger gastric pressure contractions can be clearly differentiated from the superimposed small respiratory waves. The motor inhibition due to infusion of Cecekin was a consistent response, whereas the effect of secretin varied from subject to subject as demonstrated in these two tracings. The results of the studies are discussed below.

MEAN PRESSURE Secretin produced an initial increase in mean pressure in five of eight subjects. This was followed in four of the five by a decrease in pressure after a latent period of several minutes. Cecekin always produced a prompt reduction in mean

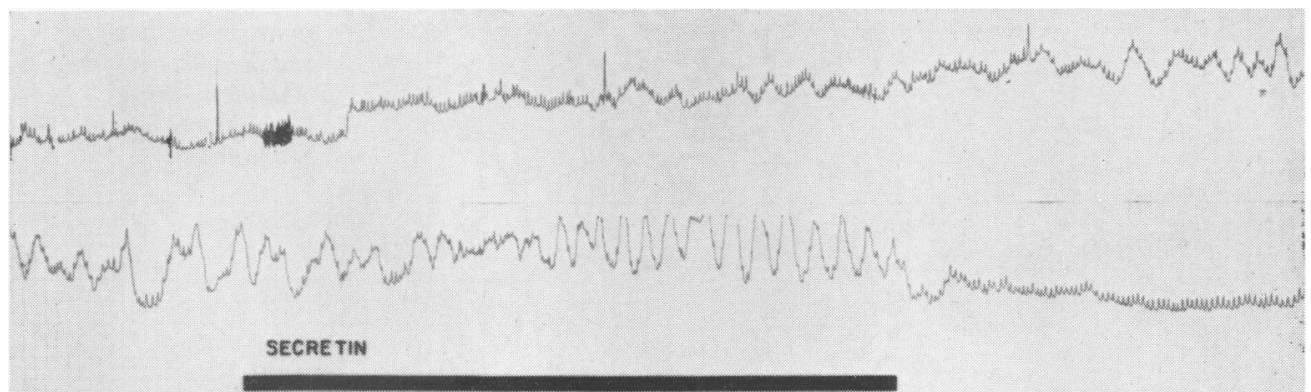

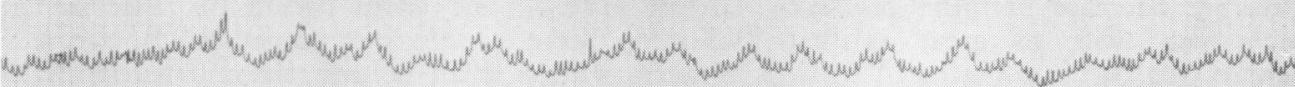
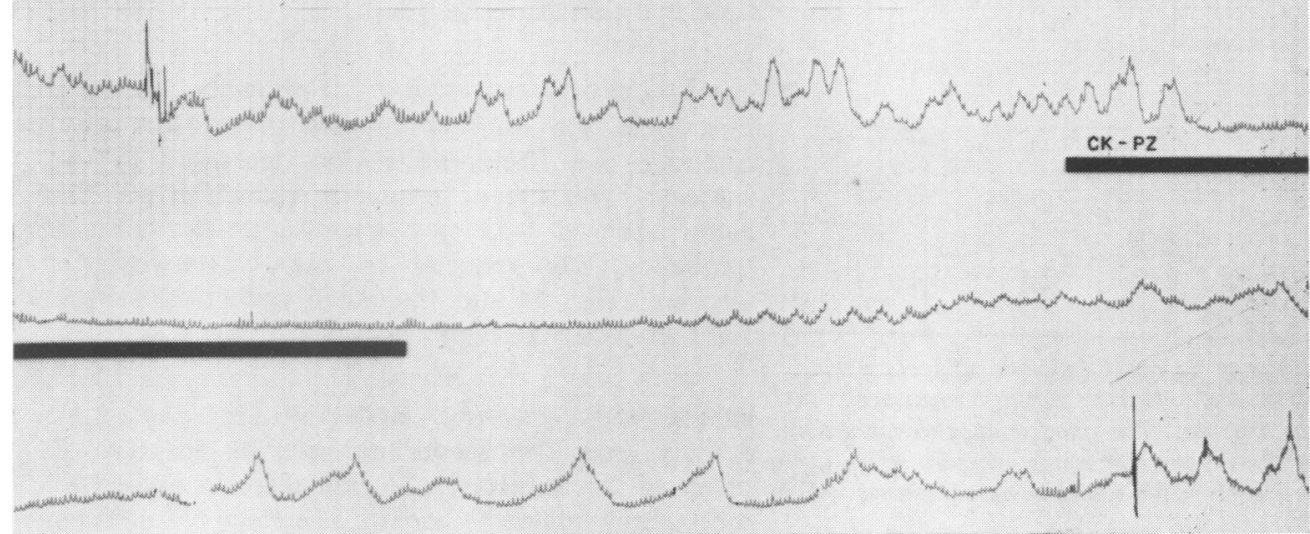

FIG. 1.

Gastric

pressure

tracing, J.M.

Each strip

represents 20

minutes of

activity.

Sharp spikes

reflect sudden

movement or

coughing.

Baseline

pressure and

wave

amplitude rise

during

secretin

injection, the

latent period

before

inhibition

being nearly

10 minutes;

subsequently

reduction in

activity with

gradual

resumption

of pressure

waves.

Cecekin

(CK/PZ)

produces

inhibition

without prior

increase in

pressure. 


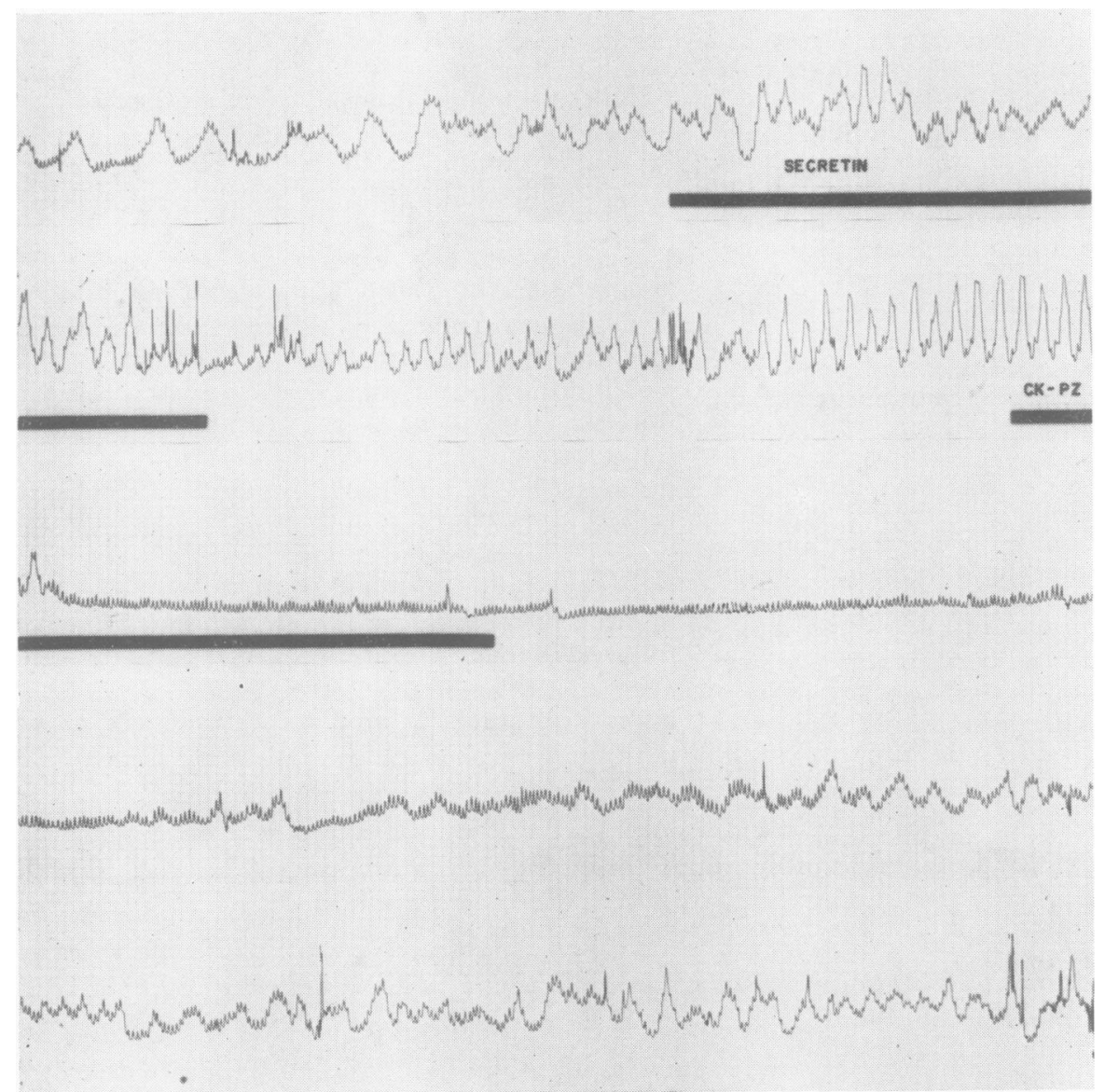

FIG. 2. Gastric pressure tracing, R.L., 100 minutes of record. The secretin infusion produced an apparent change in the pressure waves within three to four minutes, but never complete inhibition.

The variability in amplitude, frequency, and duration of the complex wave forms illustrates the difficulty in meaningful assessment of the results of secretin infusions.

The increase in baseline pressure and amplitude did not occur as in the previous figure. $B y$ contrast, the prompt and complete motor inhibition during Cecekin infusion is again noted.

pressure. The average decrease in mean pressure following Cecekin was twice as great as that produced by secretin (Fig. 3).

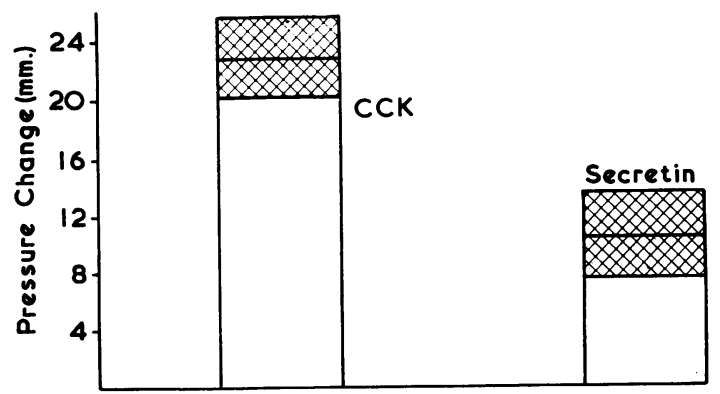

FIG. 3. Mean pressure decrease in eight subjects during maximum inhibition of gastric motility measured in millimetres on the tracings. The shaded area denotes the standard error of the mean. The mean pressure reduction was significantly greater with Cecekin as compared with secretin $(p<0.001)$.
LATENT PERIOD The latent period for inhibition following secretin infusions extended up to 10 minutes, with a mean of over six minutes in the cases in which activity was inhibited. By contrast, the latent period following Cecekin averaged less than two minutes (Fig. 4).

CONTRACTION FREQUENCY Following secretin injections, the number of gastric pressure contractions per 10-minute period decreased in five subjects. The mean frequency reduction for the eight subjects was not significant. By contrast frequency was reduced in each instance after Cecekin (Fig. 5) and the mean reduction for the eight subjects was highly significant.

PERCENTAGE ACTIVITY Reduction in percentage activity over the 10-minute interval occurred in seven of the subjects following secretin and in all the experiments with Cecekin. The mean reduction in 


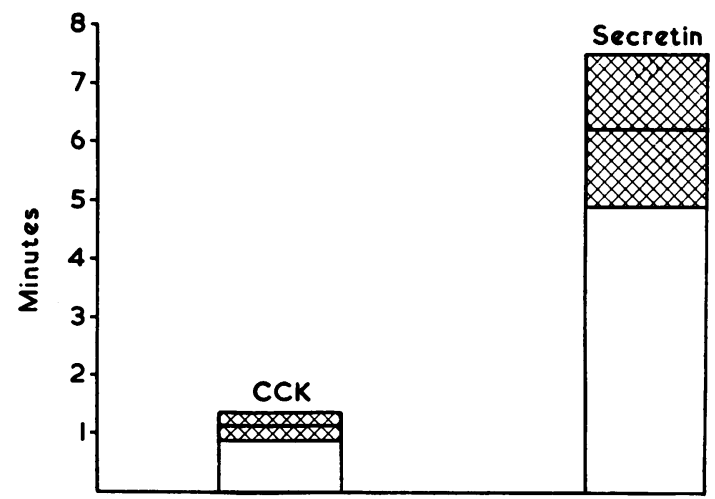

FIG. 4. Mean latent period in minutes from onset of infusion to beginning of motor inhibition in eight subjects. Again standard error is indicated by the shaded portion above and below the mean. Note the wide variation and relatively long latent period of the secretin infusions, compared with the rapid more uniform response to Cecekin. The difference betweeen the latent periods is significant. $(p<0.001)$.

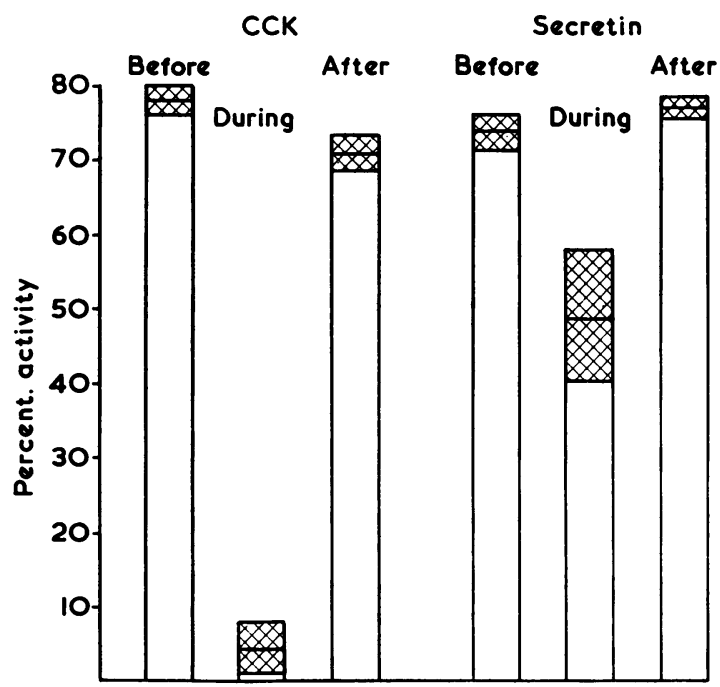

FIG. 6. Mean percentage activity in eight subjects before, during maximum inhibition, and following return of gastric motility. Percentage activity represents the fraction of the 10-minute period during which the active wave motion is apparent. Means and standard errors are denoted as previously. Note the marked reduction with Cecekin, a significant change $(p<0.001)$. Mean percentage activity with secretin infusion was perhaps significantly reduced in the eight experiments $(p<0.05)$.

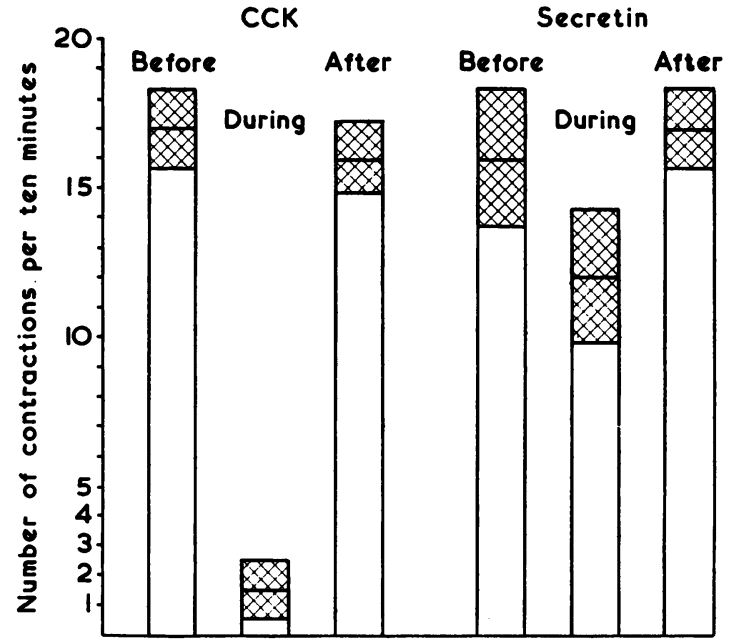

FIG. 5. Mean frequency in number of contractions per 10-minute period before, during maximum inhibition, and after recovery of activity. Again the shaded areas represent the standard error about the mean. Note the reduction in mean frequency of contractions with both Cecekin and secretin. The response to secretin, however, was not consistent, was more variable in extent, and the mean reduction was much less than with Cecekin. Comparison of the decrease from control frequency reveals significance only following Cecekin $(p<0.001)$. Mean frequency reduction due to secretin was not significant with $p<0 \cdot 1$.

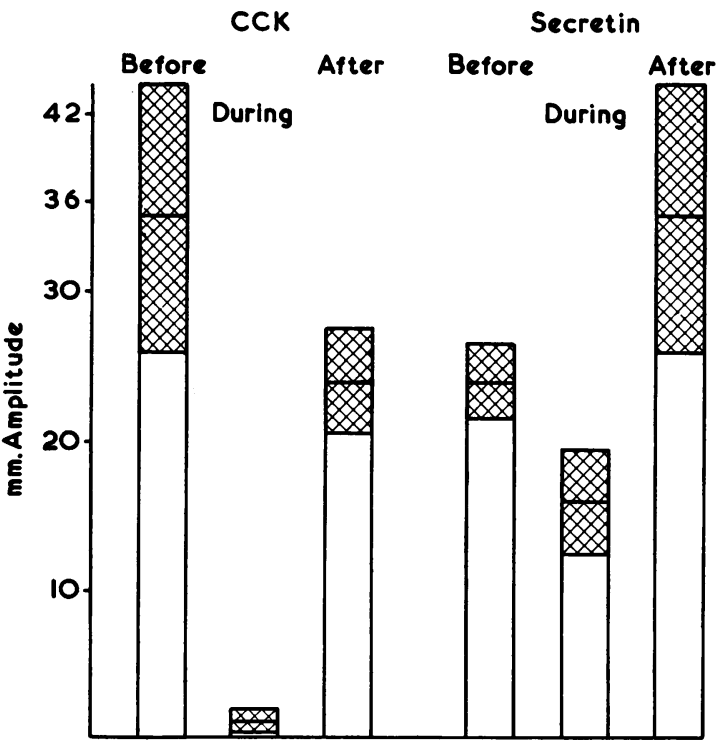

FIG. 7. Mean amplitude index, which is the summation of amplitude of all waves within the 10-minute period, whether during the control period, during maximum inhibition, or after resumption of activity. The wide variation in total amplitude is apparent by the large standard errors in the shaded portions on either side of the mean. Marked reduction occurred with Cecekin infusion $(p<0.01)$ but that due to secretin infusion was of questionable significance $(p>0.05)$. 
percentage activity was conclusively significant only in the latter group (Fig. 6).

AMPLITUDE INDEX Six of the eight tests with the secretin preparation demonstrated some reduction of amplitude index. However, the mean decrease was of borderline significance. Decreases occurred in each of the studies following Cecekin with a significant mean reduction in amplitude index for the entire group (Fig. 7).

DURATION OF INHIBITION Assessment of duration of inhibition necessarily depends on the definition of significant motor inhibition. Following secretin infusions, one half of the subjects manifested reduced gastric motility by all four criteria, viz., reductions in mean pressure, contraction frequency, percentage activity, and amplitude index. Seven of the eight subjects demonstrated decreases in two of the four criteria. Assuming motor inhibition due to secretin infusions in these seven, one can compare the duration of effect with that of Cecekin, in which reductions occurred in all four parameters in each subject. Mean values for duration of inhibition demonstrated that the effect of Cecekin lasted three times as long as that of secretin (Fig. 8).

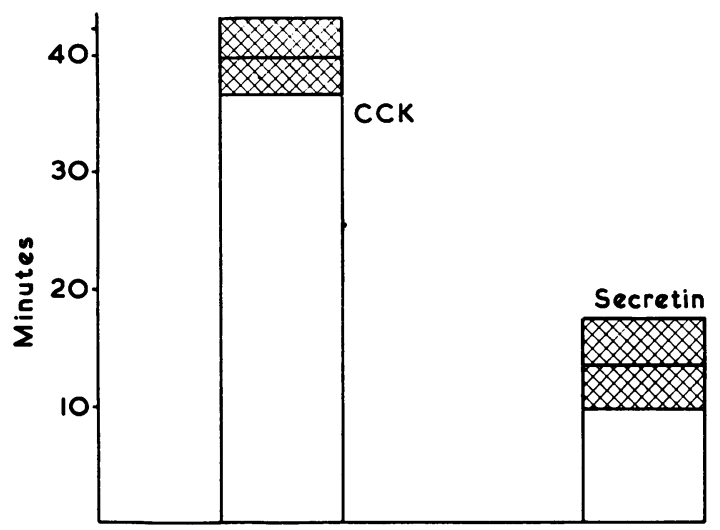

FIG. 8. Mean duration of inhibition in minutes following infusion of the two extracts. The means and standard errors are indicated. Note the prolonged effect of Cecekin compared with the relatively short period of gastric motor inhibition induced by secretin.

\section{ANCILLARY STUDIES}

Because gastric motor function following secretin preparations was not inhibited in the dog, its occurrence was surprising in man. Five of the secretin preparations used were therefore assayed for cholecystokinin activity in a conscious dog equipped with a small cholecystostomy tube (Hong,
Magee, and Crewdson, 1956). The secretin was injected intravenously in doses slightly exceeding $0.5 \mathrm{unit} / \mathrm{kg}$. None of the extracts produced a rise in intracholecystic pressure. By contrast, Cecekin consistently produced gall-bladder contraction in this animal in doses as low as $0.1 \mathrm{unit} / \mathrm{kg}$. It is concluded that the partial gastric motor inhibition produced by secretin in man cannot be explained by the presence of cholecystokinin activity in the material.

Whether the variation in response to secretin preparations was due to differences in the material (all vials used were from the same batch) or to differences in individual responsiveness, was tested in the following manner:-

One of the subjects, D.H., was noted to have rather marked inhibition following secretin. The next subject, J.A., showed very slight inhibition with secretin. The preparation which had previously caused marked inhibition of motor activity in D.H. was infused into J.A., producing only slight inhibition. This suggests individual differences rather than variation in the secretin preparation.

\section{DISCUSSION}

In man, the reduction of gastric motor activity following secretin extracts is not a consistent response, nor is the duration of inhibition comparable to that produced by the cholecystokininpancreozymin material. The induced secretin inhibition also differs from Cecekin in its comparatively long latent period. The prompt inhibition by Cecekin suggests a direct action on the gastric smooth muscle. The initial increase in contractile activity in some subjects, followed in several minutes by a reduction in motility, may represent an indirect effect in the case of secretin. Perhaps it acts secondarily by changing the content and $p \mathrm{H}$ of the duodenum through secretion of alkaline pancreatic juice. At present the mode of action of these extracts on gastric motor activity is unknown.

The consistent gastric motor inhibition in man, produced by extracts containing both cholecystokinetic and pancreozymin activity, parallels that seen in the dog. Similar preparations have been found to inhibit gastric acid secreted in response to all types of acid stimuli (Gillespie and Grossman, 1964; Jordan and De la Rosa, 1965). This material is thus capable of reproducing the action of fat in the duodenum, as measured by gastric motor and secretory activity. Because it possesses the physiological activity ascribed to enterogastrone, perhaps this preparation contains the true gastric chalone of duodenal origin. It may be that the same hormones 
which stimulate gall bladder contraction and pancreatic enzyme secretion also inhibit gastric motor and secretory activity.

\section{CONCLUSIONS}

Two duodenal extracts were administered intravenously to eight normal adults, in doses of 0.5 units/kg. given over a 10-minute period. Gastric motility was monitored by pressure changes within an intragastric balloon.

Secretin extracts produced variable results with an initial increase in intragastric pressure and motor activity, followed by subsequent clear inhibition in four subjects. In others slight or no inhibition occurred. Statistical evaluation of the secretin group revealed some inhibition of motor activity in seven of the eight subjects, but in two major parameters of measurement utilized the changes were not significant and in two the reductions were of borderline significance.

Cholecystokinin-pancreozymin extracts induced prompt, complete and more prolonged gastric motor inhibition in each of the subjects. In every category of measurement used, these reductions in gastric activity were statistically significant.

The recently reported cholecystokinin-pancreozymin inhibition of gastric acid secretion coupled with its inhibition of motility, makes it apparent that this material is capable of reproducing the physiological activity of fat in the duodenum. It is speculated that this material may contain or even be enterogastrone.

\section{REFERENCES}

Farrell, J. I., and Ivy, A. C. (1926). Studies on the motility of the transplanted gastric pouch. Amer. J. Physiol., 76, 227-228.

Gillespie, I. E., and Grossman, M. I. (1964). Inhibitory effect of secretin and cholecystokinin on Heidenhain pouch responses to gastrin extract and histamine. Gut, 5, 342-345.

Greenlee, H. B., Longhi, E. H., Guerrero, J. D., Nelsen, T. S., El Bedri, A. L., and Dragstedt, L. R. (1957). Inhibitory effect of pancreatic secretion on gastric secretion. Amer. J. Physiol., 190, 396-402.

Grossman, M. I. (1950). Gastrointestinal hormones. Physiol. Rev., 30, 33-90.

Hong, S. S., Magee, D. F., and Crewdson, F. (1956). The physiologic regulation of gall bladder evacuation. Gastroenterology, 30, 625-630.

Johnson, L. P., and Magee, D. F. (1965). Cholecystokinin/pancreozymin extracts and gastric motor inhibition. Surg. Gynec. Obstet., 121, 557-562.

Jordan, P. H., Jr., and De la Rosa, C. (1965). Inhibition of gastric secretion by duodenal mucosal extracts. Ann. Surg., 160, 978-985.

Jorpes, J. E., and Mutt, V. (1959). Secretin, pancreozymin, and cholecystokinin. Their preparation and properties. Gastroenterology, 36, 377-385.

Kosaka, T., and Lim, R. K. S. (1930). On the mechanism of the inhibition of gastric secretion by fat. The role of bile and cystokinin. Chin. J. Physiol., 4, 213-220.

Wormsley, K. G., and Grossman, M. I. (1964). Inhibition of gastric acid secretion by secretin and by endogenous acid in the duodenum. Gastroenterology, 47, 72-81. 